\title{
Novel Calibration Method for the Multi-Camera Measurement System
}

\author{
Xinlei Wang* \\ Nanjing University of Information Science \& Technology, Nanjing 210044, Jiangsu, China
}

(Received July 22, 2014 : revised October 17, 2014 : accepted October 17, 2014)

\begin{abstract}
In a multi-camera measurement system, the determination of the external parameters is one of the vital tasks, referred to as the calibration of the system. In this paper, a new geometrical calibration method, which is based on the theory of the vanishing line, is proposed. Using a planar target with three equally spaced parallel lines, the normal vector of the target plane can be confirmed easily in every camera coordinate system of the measurement system. By moving the target into more than two different positions, the rotation matrix can be determined from related theory, i.e., the expression of the same vector in different coordinate systems. Moreover, the translation matrix can be derived from the known distance between the adjacent parallel lines. In this paper, the main factors effecting the calibration are analyzed. Simulations show that the proposed method achieves robustness and accuracy. Experimental results show that the calibration can reach $1.25 \mathrm{~mm}$ with the range about $0.5 \mathrm{~m}$. Furthermore, this calibration method also can be used for auto-calibration of the multi-camera measurement system as the feature of parallels exists widely.
\end{abstract}

Keywords: Calibration, Parallel lines, Vanishing line

OCIS codes : (150.1488) Calibration; (150.4232) Multisensor methods; (200.4560) Optical data processing

\section{INTRODUCTION}

In computer vision, multi-camera measurement systems have been widely utilized in various areas [1, 2], e.g., the 3D measurement, object reconstruction, object detecting and tracking, etc. Cameras in the multi-camera measurement system are fixed; therefore, the relationship between each pair of cameras is constant. One simple but typical kind of multi-camera measurement system is the stereo measurement system, which consists of two cameras with a common field of view. Based on the point pairs, the information about the point in the scene can be obtained from many limitations, such as epipolar constraint, etc. As a well-developed theory, the stereo measurement system has been widely utilized in many industrial areas. On the other hand, the cameras in the multi-camera measurement system are not always with a common field of view according to different tasks. Therefore, when the cameras in the system have no common field of view, it is called a non-overlapping multiple vision system. Although the non-overlapping multiple vision system has no common field of view, the range of the measurement is larger. The most important task is the calibration of cameras in the multicamera measurement system, i.e. obtaining the relationship between each two cameras.

The approaches to obtaining the relationship between cameras, which is called the calibration of the external parameters of the system, are various. These approaches can be classified into two categories according to the target which is used: one uses the exact coordinates of the feature points while the other relies on the geometric or algebraic properties.

In [3], M. Knorr presented an approach to calibrate the extrinsic parameters of a multi-camera system using a ground plane, from which the homographies can be deduced. As a natural reference object, the ground plane can be estimated using a single iterated extended Kalman filters. Obviously, the relationship between each pair of cameras can be obtained. In [4], J.P. Barreto et al. estimated the extrinsic parameters of the system by moving an $L E D$ in thousands of random positions in front of cameras, and then the correspondences between views can be obtained. The projection matrices and radial distortion parameters are simultaneously computed using a factorization approach.

\footnotetext{
*Corresponding author: paper_wy@163.com

Color versions of one or more of the figures in this paper are available online.
} 
The approximate solution of a matrix is projected to the manifold of the parameter space to modify. In [5], with the help of a mirror, a common calibration object can be seen directly through all the non-overlapping view cameras. By formulating constraints between cameras and the mirrored calibration object, the extrinsic parameters can be deduced simply. For the purpose of overcoming the shortcomings of view angle, a calibration method using a non-planar target is presented in [6]. As the projection of a sphere is not related to its orientation, a target consisting of several spheres is used to finish the task of calibration. As the coordinates of the spheres can be obtained under each coordinate system, the relation is easily confirmed.

In recent years, there are more and more calibration methods which are based on the geometric features, such as the feature of a straight line. In [7], a spot laser projector was utilized to obtain two collinear feature points, which are located in different fields of view. The feature points are the cross points of the target plane and the light beam. More feature points can be obtained by moving the spot laser to different positions. Based on the constraint of the straight line, and the internal relationship between the image plane and the target plane, the calibration of the multi-camera system can be carried out easily.

All the calibration methods described above can complete the task, but in the process, the image of the feature point is necessary, so the calibration result will always be affected by the accuracy of the feature point.

In this paper, a new calibration method based on the theory of vanishing point and vanishing line, which are important features in geometry, is proposed. At least three equally spaced parallel lines are required, whether from an artificial target or a natural scene, to obtain the vanishing line of the target. Then the corresponding normal vector can be deduced. When the normal vector in each camera coordinate system is obtained, i.e. the same vector in different coordinate systems, the rotation matrix between the two coordinate systems can be easily derived. As the distance between each pair of adjacent parallel lines is known exactly, the translation matrix can be obtained. In our calibration method, all the points in the view field of each camera can be utilized to calculate the vanishing line, so our proposed method achieves robustness. And also, the feature used in our calibration method is easily obtained, whether from the artificial target or the natural scene. On the other hand, the proposed calibration method can be used to self-calibrate.

\section{PRINCIPLE}

As the stereo measurement system can be treated as a special one of the multi-camera system, in our approach, we assume two cameras located in two different positions with non-overlapping view field.

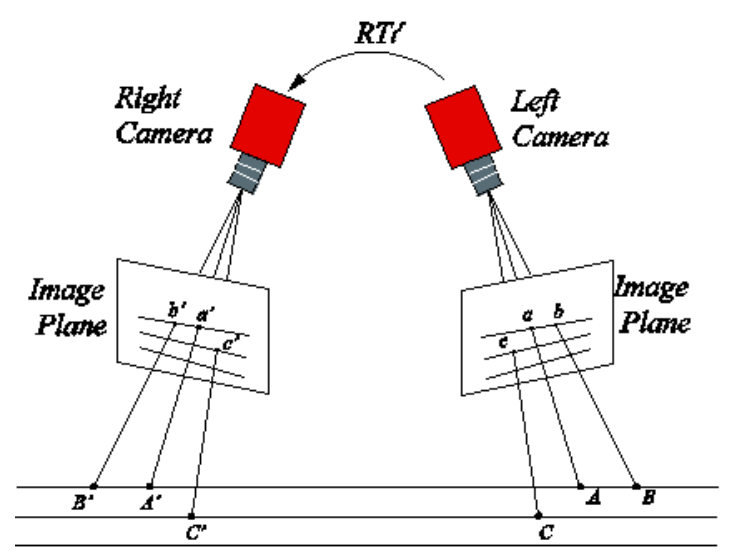

FIG. 1. The structure of the calibration system.

The structure of the measurement system is illustrated in Fig. 1. $O_{1}-X_{1} Y_{1} Z_{1}$ is the coordinate system of the left camera while $O_{\mathrm{r}}-X_{\mathrm{r}} Y_{\mathrm{r}} Z_{\mathrm{r}}$ is the right coordinate system of the right camera. $O_{1}-X_{1} Y_{1} Z_{1}$ is also treated as the global camera coordinate system and $R T_{l}^{r}$ is the notation of the transformation matrix from $O_{1}-X_{1} Y_{1} Z_{1}$ to $O_{\mathrm{r}}-X_{\mathrm{r}} Y_{\mathrm{r}} Z_{\mathrm{r}}$.

The planar target used in our calibration method is required to be at least three parallel lines with equal space. The planar target should be located in the view field of each camera. When the target is formed into a rectangle, it is easy to satisfy this requirement. The images by each camera can be captured simultaneously.

The main steps of our calibration method are listed as follows:

1) The intrinsic parameters of each camera can be calculated by Zhang's calibration method $[8,9]$. The relative position between each two camera is unchangeable while the cameras are fixed in the proper position due to the requirement of the measurement.

2) The planar target with three equally space parallel lines is used to finish the calibration. Put the target in the view field of the two cameras simultaneously (as illustrated in Fig. 1).

3) Capture the image of the target from each camera separately, and then obtain the normal vector of the target plane in each camera coordinate system. So the rotation matrix of the two camera coordinate systems can be derived from the different expressions of the same vector, i.e. the normal vector of the target plane.

4) As the distance between each pair of adjacent parallel lines is known exactly, we can get some information about the translation matrix. When the target is moved into several different positions, enough consistency can be confirmed to calculate the translation matrix.

\subsection{Related Theories}

A set of parallels in 3D space projects on the image plane of perspective geometry and the intersection is called 
the vanishing point. The vanishing point depends only on the direction of the line rather than on its position. The vanishing point can be obtained by intersecting the image plane with a ray parallel to the line and passing through the origin of the camera coordinate system. By definition and using the principle of perspective geometry, we have the following property when the direction vector of the line is defined as $\vec{n}_{L}$

$$
\vec{n}_{L}=K^{-1} V
$$

where $K$ is the intrinsic parameters matrix of the camera and $V$ is the vanishing point of the line.

In [10], a new approach of calculating the vanishing line from three coplanar equally spaced parallel lines is proposed. When the homogeneous coordinates of the three parallel lines on the image plane are defined as $l_{0}, l_{l}, l_{2}$, the vanishing line of the plane can be confirmed from the following equation:

$$
l=\left(\left(l_{0} \times l_{2}\right)^{T}\left(l_{1} \times l_{2}\right)\right) l_{1}+2\left(\left(l_{0} \times l_{1}\right)^{T}\left(l_{2} \times l_{1}\right)\right) l_{2}
$$

Moreover, we can obtain the relationship between the vanishing line and the normal vector of its corresponding plane:

$$
l=K^{-T} \vec{n}
$$

where $K$ is the intrinsic parameters matrix of the camera, and $l$ is the vanishing line while $\vec{n}$ is the normal vector of its corresponding plane.

\subsection{Obtaining the Rotation Matrix}

From the knowledge above, the normal vector of the target in the coordinate system of each camera can be derived from Eq. (3). Now the rotation matrix of the two coordinate systems can be obtained from the corresponding normal vectors using the method mentioned in $[11,12]$.

\subsection{The Confirmation of the Translation Matrix}

The normal vector of the target plane is known as $\vec{n}$ and the direction vector of the parallel lines is $\vec{n}_{L}$, which is derived in Section 2.1. Furthermore, the perpendicular vector of the parallel lines on the target plane can be confirmed from the cross of the two vectors:

$$
\vec{n}_{T}=\operatorname{cross}\left(\vec{n}, \vec{n}_{L}\right) / \operatorname{norm}\left(\operatorname{cross}\left(\vec{n}, \vec{n}_{L}\right)\right)
$$

As illustrated in Fig. 2, three random points locating in two parallel lines are point $A$ point $B$ and point $C$, their corresponding image points are assumed as point $a$ point $b$ and point $c$.

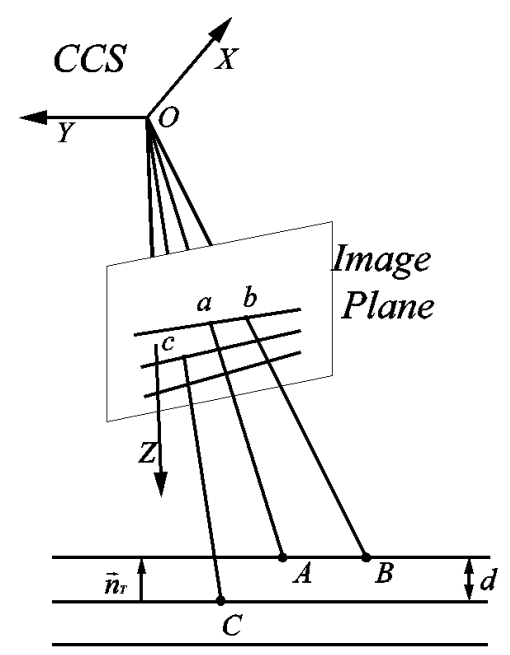

FIG. 2. The relationship between the points in the camera coordinate system.

So the relationship between the image coordinate system and the camera coordinate system is:

$$
Z\left[\begin{array}{l}
x \\
y \\
1
\end{array}\right]=\left[\begin{array}{ccc}
f_{0} & 0 & 0 \\
0 & f_{0} & 0 \\
0 & 0 & 1
\end{array}\right]\left[\begin{array}{l}
X \\
Y \\
Z
\end{array}\right]
$$

where $f_{0}$ is the effective focal length $(E F L)$, and $\left[\begin{array}{lll}x & y & 1\end{array}\right]$ is the image point of the point $\left[\begin{array}{lll}X & Y & Z\end{array}\right]$ under the camera coordinate system.

Obviously, the direction vector from point $A$ to point $B$ is the direction vector of the parallel lines, i.e.

$$
\overrightarrow{A B}=\vec{n}_{L}
$$

The projection of the vectors $\overrightarrow{A C}$ and $\overrightarrow{B C}$ onto the vector $\vec{n}_{T}$, which is the vertical vector of the parallel lines, is the distance $d$ between each pair of adjacent parallel lines. As the vector $\vec{n}_{T}$ is a unit vector, we have the relationship as below:

$$
\left\{\begin{array}{l}
\operatorname{dot}\left(\overrightarrow{A C}, \vec{n}_{T}\right)=d, \\
\operatorname{dot}\left(\overrightarrow{B C}, \vec{n}_{T}\right)=d,
\end{array}\right.
$$

Combined Eq. (5) with Eq. (6) and Eq. (7), the coordinates of the points $A, B$ and $C$ can be derived. Moreover, the coordinates of the point $A$ and the point $B$, which are two random points in the left camera coordinate system, and the coordinates of the point $A^{\prime}$ and the point $B^{\prime}$, which are two random points in the right camera coordinate system, can be obtained in the corresponding camera coordinate system. 
Point $A$ and point $B$ can be transformed to the right camera coordinate system according to the constraint of the collineation. So the relation can be expressed as

$$
\overrightarrow{A^{\prime} B^{\prime}}=\overrightarrow{A B^{\prime}}
$$

When the target is moved into more than two different positions, more equations like Eq. (8) can be obtained, from which we can derive the translation matrix simply.

\section{SIMULATIONS AND DISCUSSIONS}

Simulations will be conducted to evaluate the impacts of some vital factors. The assumed multi-camera measurement system consists of two identical cameras, and the intrinsic parameters of each camera, which are listed in Table 1, are also identical.

TABLE 1. The intrinsic parameters of the camera

\begin{tabular}{c|c|c|c|c}
\hline \hline$f_{0}(\mathrm{~mm})$ & $f_{x}$ (pixels) & $f_{y}$ (pixels) & $u_{0}($ pixels $)$ & $v_{0}$ (pixels) \\
\hline 16 & 3953.4 & 3949.3 & 863.7 & 515.7 \\
\hline
\end{tabular}

where $f_{0}$ is the effective focal length, $f_{x}$ is the scale factor in the $x$-coordinate direction, $f_{y}$ is the scale factor in the $y$-coordinate direction, $\left(u_{0}, v_{0}\right) \mathrm{T}$ are the coordinates of the principal point. Now the simulations are detailed below.

\subsection{The Number of Target Positions}

In the proposed method, one camera can capture one image of the target when the target is located in one position. And the normal vector of the target plane can be confirmed based on the property of the vanishing line. Theoretically, the rotation matrix can be determined by two vector pairs. But as the noise is inevitable, the normal will involve additive error. If more vector pairs can be used to confirm the rotation matrix, the additive error will be diminished. For the translation matrix, the number of target positions is the main factor, which can be obtained from the confirmation of the translation matrix. Therefore, the number of target positions is the only factor related to the translation matrix in this paper.

For the purpose of verifying the affection of the number of target positions, we conducted a simulation. In the simulation, the distance of each two adjacent lines is 20 $\mathrm{mm}$, and the Gaussian noise with mean 0 and standard deviations of 0.5 pixels is added to the captured images. The number of target positions is varied from 3 to 10 . In order to make the illustration clear, we transform the rotation matrix to the rotation vector and then calculate the angle between the ideal vector and the obtained one. The Root-Mean-Square $(R M S)$ error and the mean absolute error $(M A E)$ as the function of the number of target positions are illustrated in Fig. 3(a). For the translation matrix,

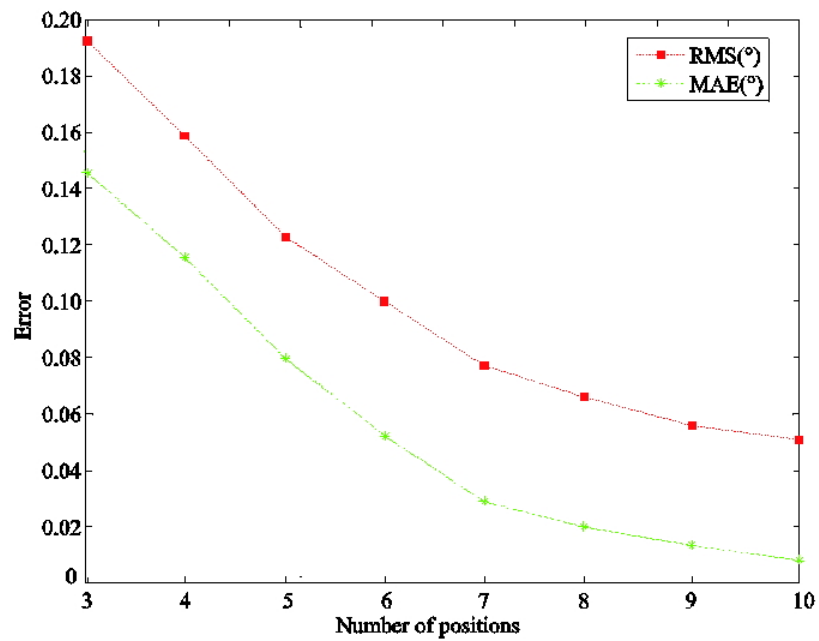

(a)

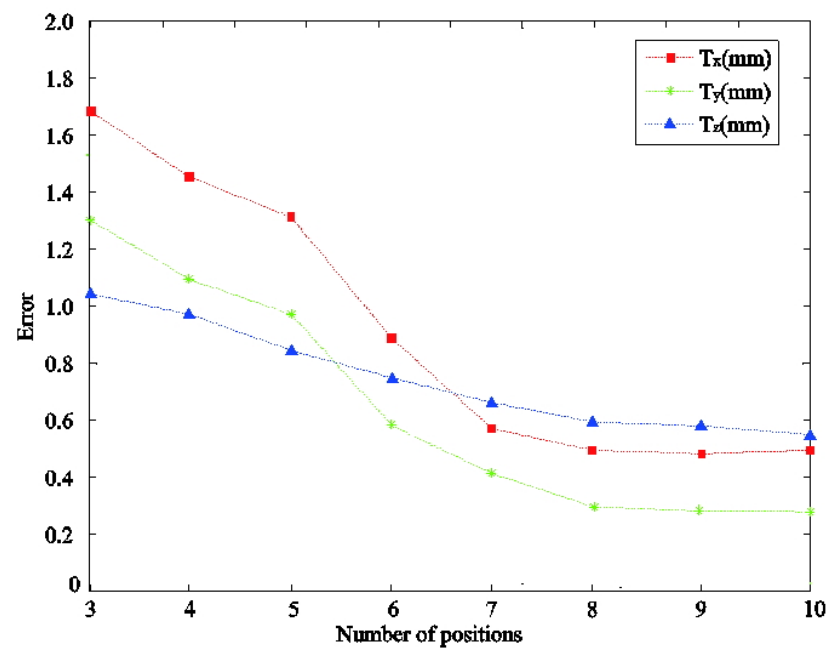

(b)

FIG. 3. (a) The $R M S$ error and the $M A E$ of the rotation matrix as the function of the number of target positions; (b) The $M A E$ of the translation matrix.

which is defined as $\left[\begin{array}{lll}T_{\mathrm{x}} & T_{\mathrm{y}} & T_{\mathrm{z}}\end{array}\right]^{T}$, the MAEs of the three elements are calculated, and the result is illustrated in Fig. 3(b). Each point in Fig. 3 represents the result averaged over 300 uniformly distributed rotations.

It is obvious that the RMS error and the $M A E$ decline as the number of the target position increases, which means that the error of the rotation matrix is decreasing. The similar situation still can be found in the determination of the translation matrix. Therefore, if possible, the target should be located in different positions to make the calibration more accurate.

\subsection{The Distance between Two Adjacent Parallel Lines}

As it has been known, the normal vector of the target plane is determined by the parallel lines on the target. Nevertheless, when the distance between two adjacent parallel lines is large, the representation of the feature of vanishing 


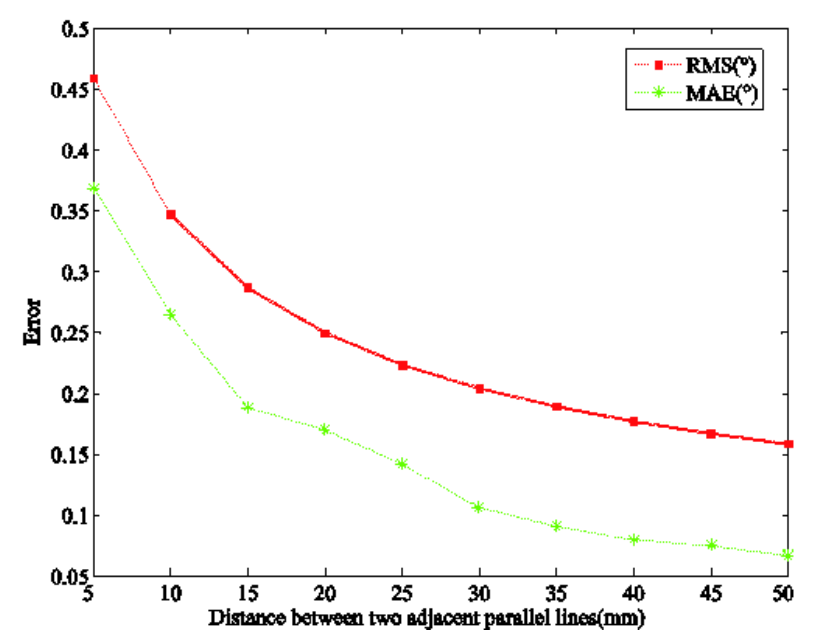

FIG. 4. The RMS error and the $M A E$ of the angle as the function of the distance between the two adjacent parallel lines.

is notable. In this case, the effect of the additive noise will be decreased. A simulation will be conducted to verify the conclusion. Similarly, the Gaussian noise with mean 0 and standard deviation of 0.5 pixels is added to the idealized ones to generate the perturbed images. The number of target positions in the simulation is 5 . The distance between adjacent parallel lines varies from $5 \mathrm{~mm}$ to $50 \mathrm{~mm}$ with the interval of $5 \mathrm{~mm}$. The criterion of the simulation is the same as mentioned in Section 3.1, the angle between the idealized rotation vector and the obtained one is used to measure the error involved in the determination of the rotation matrix. The RMS error and the $M A E$ of the angle as a function of the distance between the two adjacent parallel lines are illustrated in Fig. 4.

As illustrated in Fig. 4, the angle decreases as the distance between the two adjacent parallel lines increases. So it is appropriate to increase the distance if it is possible. Meanwhile, the distortion of the lens cannot be neglected, if possible, the parallel lines should form an image in the center of the image plane, while the increasing of the space may make it more difficult.

\subsection{The Number of Points Used to Determine the Parallel Line}

Obviously, the normal vector is one of the vital factors affecting the rotation matrix directly, while the normal vector is determined by the extraction of the parallel lines. In the proposed method, the extraction of the center of the line is based on C.Steger's method mentioned in [13], which has the capacity of reaching sub-pixel accuracy. Then the function of the line can be determined by the fitting of the separate points. So the number of image points is another important factor affecting the calibration result. For this purpose, the related simulation has been conducted to evaluate the situation. In this simulation, the number of points used to fit each parallel line varies from 100 to 1000 points with the interval of 100 points. The

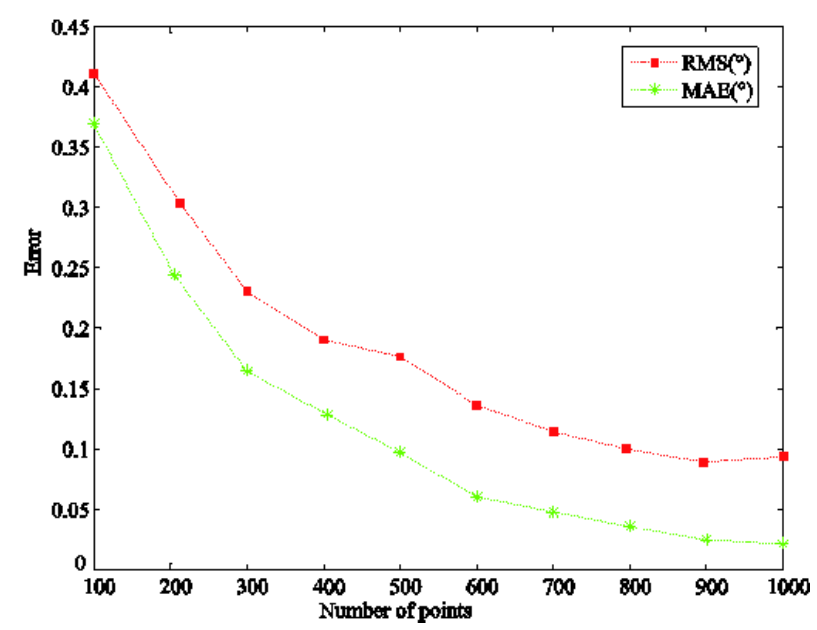

FIG. 5. The $R M S$ error and the $M A E$ as the function of the number of points.

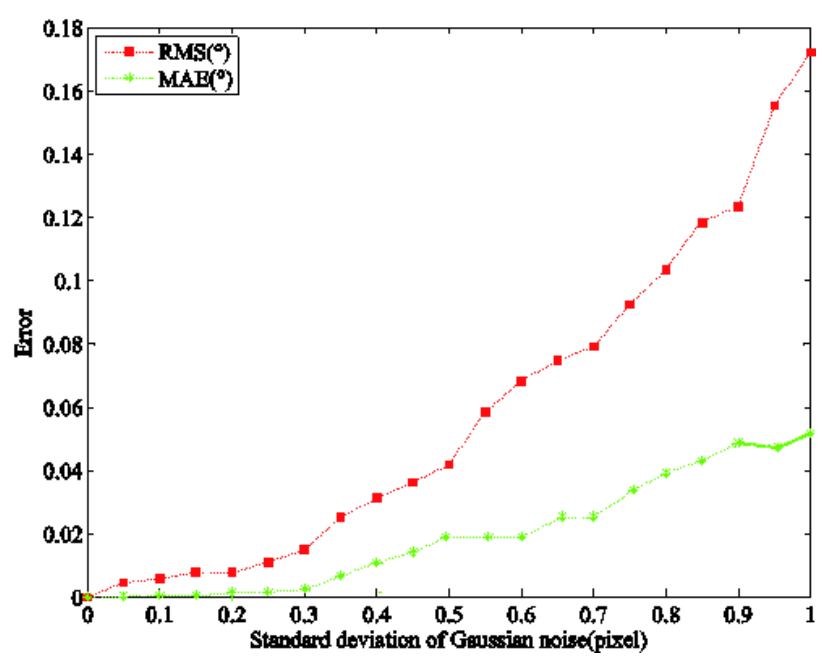

FIG. 6. The calibration result with different additive noise.

distance between each pair of adjacent parallel lines is 20 $\mathrm{mm}$, while the number of target positions is 5. The criterion evaluating the calibration results is the same as the description mentioned in Section 3.1, and the angle between the ideal rotation vector and the obtained one is the target. The related result is illustrated in Fig. 5.

\subsection{The Total Simulation}

For the purpose of evaluating the practicability of the proposed method, the simulation will be conducted. Gaussian noise with different standard deviations is added to the ideal image to generate a perturbed one. The distance between the two adjacent parallel lines is $20 \mathrm{~mm}$, while the number of target positions is 5. The rotation matrix is still evaluated by the RMS error of the angle between the ideal rotation vector and the obtained one. The translation matrix is evaluated by the absolute bias of each element. The calibration results are plotted in Fig. 6 . 
TABLE 2. The intrinsic parameters of the two cameras

\begin{tabular}{c|c|c|c|c|c|c}
\hline \hline Parameters & $f_{\mathrm{x}}$ (pixels) & $f_{y}$ (pixels) & $u_{0}$ (pixels) & $v_{0}$ (pixels) & $k_{1}$ & $k_{2}$ \\
\hline Left camera & 5125.2 & 5124.9 & 1270.7 & 1037.7 & -0.2531 & 0.3777 \\
\hline Right camera & 5012.4 & 5011.7 & 1201.3 & 1015.7 & -0.2310 & 0.5276 \\
\hline
\end{tabular}

TABLE 3. Experimental result of the repeatability

\begin{tabular}{c|c|c|c|c}
\hline \hline Parameters & Angle $\left({ }^{\circ}\right)$ & $\mathrm{T}_{\mathrm{x}}(\mathrm{mm})$ & $\mathrm{T}_{\mathrm{y}}(\mathrm{mm})$ & $\mathrm{T}_{\mathrm{z}}(\mathrm{mm})$ \\
\hline$R M S$ error & 0.021 & 0.501 & 0.469 & 0.175 \\
\hline
\end{tabular}
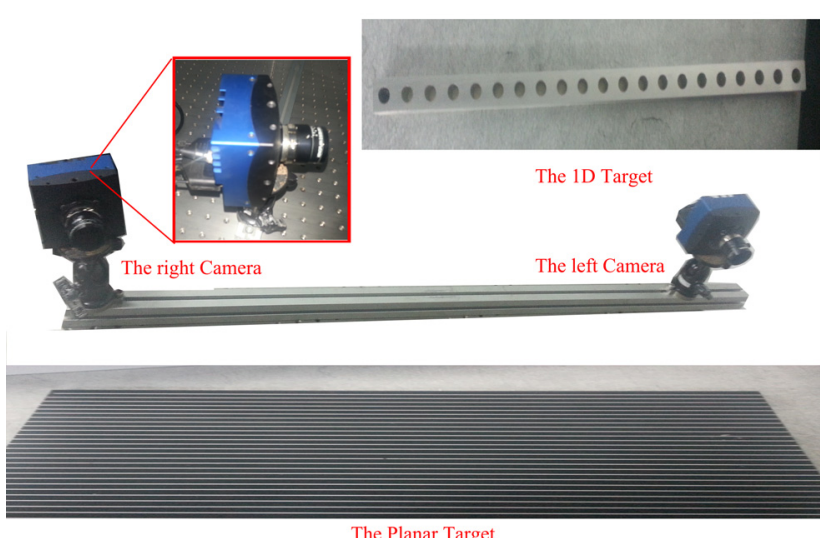

FIG. 7. The construct of the real experiment.

\section{THE REAL EXPERIMENT AND DISCUSSIONS}

In the real experiment, the multi-camera measurement system consists of two cameras, which are Mikrotron MC4082's with a resolution of $2336 \times 1728$ pixels. The intrinsic parameters of each camera, listed in Table 2, are obtained by Zhang's method. The notations $f_{x}, f_{y}, u_{0}, v_{0}$ are defined as mentioned in Table 1 , while $k_{1}, k_{2}$ are the radial distortion coefficients of the lens.

The target used in our experiment is a planar target with a series of parallel lines on it (Fig. 7), but actually, only three equally space parallel lines are used to determine the vanishing line, and further, the normal vector of the target plane.

Then the rotation matrix can be confirmed, while the translation matrix is determined by the steps described in section 2. According to the proposed method, the rotation matrix we obtained is $\left[\begin{array}{ccc}0.541 & 0.624 & -0.564 \\ 0.838 & -0.461 & 0.293 \\ -0.077 & -0.634 & -0.772\end{array}\right]$, while the translation matrix is $\left[\begin{array}{c}-60.01 \\ 10.46 \\ 591.54\end{array}\right]$.

\subsection{The Experiment for Repeatability}

In order to verify the repeatability of the proposed method, we calibrate the multi-camera measurement system 8 times. In each time, the same experiment condition is requested. The standard deviation of the rotation vector and the translation vector are calculated, and the result is listed in Table 3.

\subsection{The Experiment for Determining the Accuracy}

For the purpose of evaluating the accuracy of the proposed method, the method mentioned in Ref. [7] is used. Similarly, a 1D target with several feature points at each side is utilized (Fig. 7). The feature points on the left side are captured by the left camera while the feature points on the right side are captured by the right camera. As the constraint of the length, the coordinates of the feature points in each camera coordinate system can be determined. Then the feature points can be transformed to the right camera coordinate system according to the calibration result. The distance between the feature point captured by the left camera and the feature point captured by the right camera can be derived, while it is known exactly beforehand. The results are listed in Table 4.

$\left[X_{\mathrm{r}}, Y_{\mathrm{r}}, Z_{\mathrm{r}}\right]^{\mathrm{T}}$ in the table is the coordinate of the feature point in the right camera coordinate system while the $\left[X_{1}, Y_{1}, Z_{1}\right]^{\mathrm{T}}$ is the coordinate of the feature point which is captured by the left camera but transformed to the right camera coordinate system. The notation $d \mathrm{~m}$ is the measured distance while the notation $d \mathrm{~s}$ is the sign of the standard length.

\section{CONCLUSION}

In this paper, a new calibration method for the external parameters of the multi-camera measurement system is proposed. Using a target with three equally spaced parallel lines, the normal vector of the target plane can be confirmed. By moving the target into at least three different positions, the rotation matrix can be determined. As the distance between two adjacent parallel lines is known exactly, the translation matrix can also be obtained. Then all the external parameters are confirmed. The simulations 
TABLE 4. The accuracy test for the calibration result

\begin{tabular}{c|c|c|c|c|c|c|c|c|c}
\hline \hline NO. & $\mathrm{X}_{1}(\mathrm{~mm})$ & $\mathrm{Y}_{1}(\mathrm{~mm})$ & $\mathrm{Z}_{\mathrm{l}}(\mathrm{mm})$ & $\mathrm{X}_{\mathrm{r}}(\mathrm{mm})$ & $\mathrm{Y}_{\mathrm{r}}(\mathrm{mm})$ & $\mathrm{Z}_{\mathrm{r}}(\mathrm{mm})$ & $\mathrm{d}_{\mathrm{m}}(\mathrm{mm})$ & $\mathrm{d}_{\mathrm{s}}(\mathrm{mm})$ & $\Delta \mathrm{d}(\mathrm{mm})$ \\
\hline 1 & -247.2 & 158.7 & 400.2 & -156.4 & 249.5 & 672.7 & 301.3 & 300 & 1.3 \\
\hline 2 & -247.2 & 158.7 & 400.2 & -150.4 & 255.5 & 690.7 & 321.2 & 320 & 1.2 \\
\hline 3 & -247.2 & 158.7 & 400.2 & -144.3 & 261.6 & 708.8 & 341.2 & 340 & 1.2 \\
\hline 4 & -247.2 & 158.7 & 400.2 & -138.3 & 267.6 & 726.8 & 361.1 & 360 & 1.1 \\
\hline 5 & -247.2 & 158.7 & 400.2 & -132.2 & 276.7 & 745.1 & 381.3 & 380 & 1.3 \\
\hline 6 & -247.2 & 158.7 & 400.2 & -126.2 & 279.7 & 763.1 & 401.2 & 400 & 1.2 \\
\hline 7 & -207.7 & 138.1 & 366.9 & -121.3 & 259.0 & 626.0 & 298.7 & 300 & -1.3 \\
\hline 8 & -207.7 & 138.1 & 366.9 & -115.5 & 267.2 & 643.5 & 318.9 & 320 & -1.1 \\
\hline 9 & -207.7 & 138.1 & 366.9 & -109.7 & 275.3 & 660.8 & 338.8 & 340 & -1.2 \\
\hline 10 & -207.7 & 138.1 & 366.9 & -103.9 & 293.4 & 678.2 & 358.9 & 360 & -1.1 \\
\hline 11 & -207.7 & 138.1 & 366.9 & -98.1 & 291.5 & 695.6 & 378.9 & 380 & -1.1 \\
\hline 12 & -207.7 & 138.1 & 366.9 & -92.4 & 299.5 & 712.8 & 398.7 & 400 & -1.3 \\
\hline
\end{tabular}

and real experiments show that the proposed method is accurate and with good robustness. The calibration results can reach about $1.25 \mathrm{~mm}$ with the range of $0.5 \mathrm{~m}$.

Moreover, as the feature of parallel lines exists widely, the proposed calibration method can also be used for auto-calibration. For instant, the natural feature of parallel lines, such as the edges of the windows or the building, can be utilized to finish the calibration, especially when the target is not proper or for a measurement system with a wide field of view.

\section{REFERENCES}

1. M. Cho and D. Shin, "Depth resolution analysis of axially distributed stereo camera systems under fixed constrained resources," J. Opt. Soc. Korea 17, 500-505 (2013).

2. J. Hwang, S. Park, C. Park, G. Lee, and K. Kim, "A display-based visual stimulator for psychophysical and electrophysiological color sensitivity measurements," J. Opt. Soc. Korea 16, 145-150 (2012).

3. K. Moritz, N. Wolfgang, and S. Christoph, "Online extrinsic multi-camera calibration using ground plane induced homographies," in Proc. IEEE Intelligent Vehicles Symposium (Gold Coast, Australia, June 2013), pp. 236-241.

4. J. P. Barreto and K. Daniilidis, "Wide area multiple camera calibration and estimation of radial distortion," in Proc. OMNIVIS 2004 - Workshop on Omnidirectional Vision and Camera Networks (Prague, CZ, 2004).
5. R. K. Kumar, A. Ilie, J. Frahm, and M. Pollefeys, "Simple calibration of non-overlapping cameras with a mirror," in Proc. Computer Vision and Pattern Recognition (CVPR) (Anchorage, AK, 2008), pp. 1-7.

6. E. Shen and R. Hornsey, "Multi-camera network calibration with a non-planar target," IEEE Sensors Journal 11, 2356-2364 (2011).

7. Z. Liu, X. G. Wei, and G. J. Zhang, "External parameter calibration of widely distributed vision sensors with nonoverlapping fields of view," Optics and Lasers in Engineering 51, 643-650 (2013).

8. Z. Zhang, "A flexible new technique for camera calibration," IEEE Trans. Pattern Anal. Mach. Intell. 22, 1330-1334 (2000).

9. J. Bouguet, "Camera calibration toolbox for Matlab," http://www.vision.caltech.edu/bouguetj/ calib_doc/ (December 2013).

10. R. Hartley and A. Zisserman, Multiple View Geometry in Computervision (Cambridge University Press, Cambridge, UK, 2003), Chapter 2-8.

11. M. D. Shuster and S. D. Oh, "Three-axis attitude determination from vector observations," J. Guidance and Control 4, 70-77 (1981).

12. K. P. Horn, "Closed-form solution of absolute orientation using unit quaternions," Optical Society of America A 4, 629-642 (1987).

13. C. Steger, "Unbiased extraction of curvilinear structures from 2D and 3D image," $\mathrm{Ph}$. D. Dissertation, Technische Universitaet Muenchen (1998). 